\title{
Metrics and Quantification of Operational and Infrastructure Resilience in Power Systems
}

DOI:

10.1109/TPWRS.2017.2664141

\section{Document Version}

Accepted author manuscript

Link to publication record in Manchester Research Explorer

\section{Citation for published version (APA):}

Panteli, M., Mancarella, P., Trakas, D. N., Kyriakides, E., \& Hatziargyriou, N. (2017). Metrics and Quantification of Operational and Infrastructure Resilience in Power Systems. IEEE Transactions on Power Systems.

https://doi.org/10.1109/TPWRS.2017.2664141

\section{Published in:}

IEEE Transactions on Power Systems

\section{Citing this paper}

Please note that where the full-text provided on Manchester Research Explorer is the Author Accepted Manuscript or Proof version this may differ from the final Published version. If citing, it is advised that you check and use the publisher's definitive version.

\section{General rights}

Copyright and moral rights for the publications made accessible in the Research Explorer are retained by the authors and/or other copyright owners and it is a condition of accessing publications that users recognise and abide by the legal requirements associated with these rights.

\section{Takedown policy}

If you believe that this document breaches copyright please refer to the University of Manchester's Takedown Procedures [http://man.ac.uk/04Y6Bo] or contact uml.scholarlycommunications@manchester.ac.uk providing relevant details, so we can investigate your claim.

\section{OPEN ACCESS}




\title{
Metrics and Quantification of Operational and Infrastructure Resilience in Power Systems
}

\author{
Mathaios Panteli, Member, IEEE, Pierluigi Mancarella, Senior Member, IEEE, Dimitris N. Trakas, Student \\ Member, IEEE, Elias Kyriakides, Senior Member, IEEE, and Nikos D. Hatziargyriou, Fellow, IEEE
}

\begin{abstract}
Resilience to high impact low probability events is becoming of growing concern, for instance to address the impacts of extreme weather on critical infrastructures worldwide. However, there is, as yet, no clear methodology or set of metrics to quantify resilience in the context of power systems and in terms of both operational and infrastructure integrity. In this paper, the resilience "trapezoid" is therefore introduced which extends the resilience "triangle" that is traditionally used in existing studies, in order to consider the different phases that a power system may experience during an extreme event. The resilience trapezoid is then quantified using time-dependent resilience metrics that are specifically introduced to help capture the critical system degradation and recovery features associated to the trapezoid for different temporal phases of an event. Further, we introduce the concepts of operational resilience and infrastructure resilience to gain additional insights in the system response. Different structural and operational resilience enhancement strategies are then analyzed using the proposed assessment framework, considering single and multiple severe windstorm events that hit the 29-bus Great Britain transmission network test case. The results clearly highlight the capability of the proposed framework and metrics to quantify power system resilience and relevant enhancement strategies.
\end{abstract}

Index Terms - Critical Infrastructure, Extreme Weather, High Impact Low Probability Event, Power Systems Resilience, Power Systems Resiliency

\section{INTRODUCTION}

$\mathrm{T}$ HE effect of extreme weather on power systems is becoming increasingly evident worldwide in the last decades. These events are usually categorized as high-impact low-probability events, as their frequency may be relatively low, but their impact may be extremely high.

One of the first attempts for defining resilience comes from C. S. Holling in 1973 [1] for ecological systems, based on which various definitions have arisen in different disciplines,

M. Panteli is with the School of Electrical and Electronic Engineering, The University of Manchester, Manchester, M13 9PL, UK (email: mathaios.panteli@manchester.ac.uk).

P. Mancarella is with the Department of Electrical and Electronic Engineering, The University of Melbourne, Parkville, Victoria, 3010, Australia; and with the School of Electrical and Electronic Engineering, The University of Manchester, Manchester, M13 9PL, UK (emails: pierluigi.mancarella@unimelb.edu.au; p.mancarella@manchester.ac.uk)

D. N. Trakas and N. D. Hatziargyriou are with the National Technical University of Athens, Athens 157 73, Greece (e-mail: dtrakas@power.ece.ntua.gr; nh@power.ece.ntua.gr).

E. Kyriakides are with the Department of Electrical and Computer Engineering, University of Cyprus, Nicosia, 1678, Cyprus (email: elias@ucy.ac.cy).

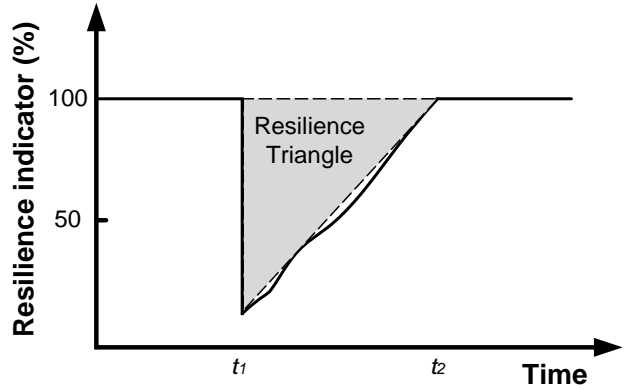

Fig. 1. The resilience triangle [2]

such as social systems [3], community [4], economy [5] and health systems [6]. Despite the minor differences between these definitions, the concept of resilience in any discipline (including power systems) can be in general approached as the ability of a system to anticipate and withstand external shocks, bounce back to its pre-shock state as quickly as possible and adapt to be better prepared to future catastrophic events. Defining and understanding power systems resilience to such catastrophic events has recently attracted the interest of several researchers [7-9]. Several resilience-oriented studies have been developed over the years [10-17], including modelling techniques and resilience enhancement strategies. Further, different from the traditional reliability indices (e.g. Loss of Load Frequency, LOLF, and Loss of Load Expectation, $L O L E$ ), various metrics have been proposed for specifically quantifying resilience. For example, resilience is quantified as the ratio of the area between the real and target performance curves in [10,11, 15]. In [12], system resilience is measured using the proportion of delivery function that has been recovered from its disrupted state, while in [13] resilience is measured as the difference between the capacity of the fully functioning system and the post-event capacity.

The so-called "resilience triangle" [2], depicted in Fig. 1, is modelled in the majority of these studies, even though different performance response curves of an infrastructure have also generally been discussed (for example in [10] and [12]). The shape of the triangle's hypotenuse can vary, e.g. linear, triangular or exponential [14], depending on the effectiveness of the recovery strategies in place. However, although this approach can effectively capture the resilience recovery following an event $\left(t \in\left[t_{1}, t_{2}\right]\right)$, it cannot capture other highly critical resilience dimensions that may be experienced by typical power systems, e.g., how fast resilience degrades once the event hits a critical infrastructure or how long the infrastructure remains in one or more post-event degraded states before restoration is initiated and while it is fully accomplished. It is therefore not capable of providing a 
complete picture of the resilience level of a critical infrastructure during all the phases of an event. Further, it does not allow the evaluation of the contribution of different adaptation and resilience enhancement strategies in each one of these phases. This is also to be seen in the light of the fact that there may be different levels of resilience that may be achieved from an operational perspective and from an infrastructure perspective, and that different options will have different effects (and costs) on both. Relevant metrics that can quantitatively capture all this complexity are also missing. Finally, the existing works mainly focus on assessing the resilience of critical infrastructures to a single weather event. However, as evidenced by recent major disasters, multiple events might hit the infrastructure in a short time period, resulting in further resilience degradation.

In this paper, a novel resilience quantification framework is proposed, building around the concept of a resilience trapezoid that depicts all the phases that a critical infrastructure, including power systems, might reside in during an event, as well as the transition between these states. Similar curves to this trapezoid have been provided in previous works (for example $[10,12,15]$ ), but have not been adequately and methodically modelled or quantified. Time-dependent operational and infrastructure resilience metrics based on different indicators are proposed in this work to quantify this multi-phase resilience trapezoid and relevant critical resilience metrics, i.e., how low and how fast resilience drops when the weather event hits a power system, how long it resides to the post-event degraded state and how fast it recovers to its preevent state, enabling the modelling of the actual, timedependent behavior of an infrastructure subject to an extreme event and making indeed a distinction between operational and infrastructure impacts. Then, building on and synthesizing these four metrics, the area of the trapezoid is also used as a complementary, quantitative metric for gaining additional information on the resilience performance of the infrastructure and the effect of various resilience enhancement strategies.

Without loss of generality, the assessment framework is exemplified considering extreme weather events ${ }^{1}$ and using fragility curves for obtaining the time- and weather-dependent failure probabilities of power system components. Several case studies are carried out on the Great Britain (GB) transmission test network [18], in order to quantify both operational and infrastructure resilience and the effect of enhancement strategies using the proposed resilience metric framework. Further, the network resilience is quantified when subject to single and multiple successive windstorms, for a better understanding of the impacts of such disastrous events.

The rest of the paper is organized as follows. Section II introduces the proposed resilience quantification framework. The fragility modelling and simulation procedure are briefly outlined in Section III. Section IV presents different case study applications using the 29-bus test version of the GB transmission network. Finally, Section V concludes the paper.

\footnotetext{
${ }^{1}$ Severe windstorms with varying intensities hitting transmission networks are used as an illustrative case study, but any (weather) event could be used if the relevant data are available.
}

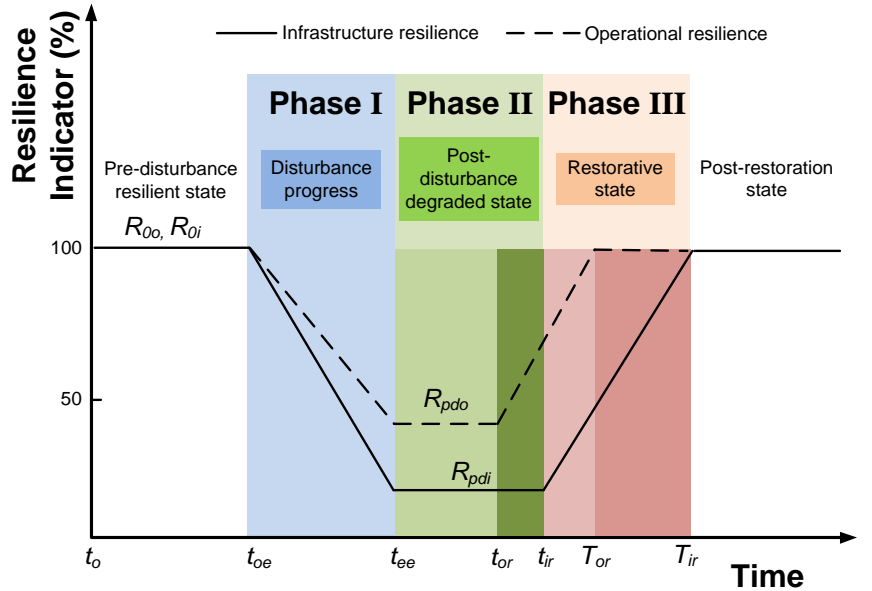

Fig. 2. The multi-phase resilience trapezoid

\section{QUANTITATIVE APPROACH AND METRICS FOR RESILIENCE ASSESSMENT OF POWER SYSTEMS}

The multi-phase resilience trapezoid is discussed in this section, along with the time-dependent resilience metrics and indicators used in this study for quantifying the operational and infrastructure resilience of a power system.

\section{A. Multi-phase resilience trapezoid associated to an event}

Fig. 2 shows the multi-phase resilience trapezoid ${ }^{2}$, where the resilience indicator used for quantifying the resilience level of a power system during the event is shown as a function of time. Further, the operational and infrastructure resilience are depicted, which, as discussed later, are quantified using different indicators. The operational resilience, as its name suggests, refers to the characteristics that would secure operational strength for a power system, e.g., the ability to ensure the uninterrupted supply to customers or generation capacity availability in the face of a disaster. The infrastructure resilience refers to the physical strength of a power system for mitigating the portion of the system that is damaged, collapsed or in general becomes nonfunctional.

In Fig. 2, it is considered that the resilience indicators used for expressing and quantifying the operational and infrastructure resilience levels in the pre-disturbance resilient state (i.e., $R_{0 o}$ and $R_{0 i}$ respectively) are $100 \%$ before the event occurs at $t_{o e}$, e.g. $100 \%$ of the demand and of the transmission lines are online respectively. However, it must be clarified that these resilience levels might differ.

Three phases can be clearly seen in the resilience trapezoid of Fig. 2, namely:

- Phase I, disturbance progress ( $\left.t \in\left[t_{o e}, t_{e e}\right]\right)$, between the time of the event $t_{o e}$ and the end of the event $t_{e e}$,

Phase II, post-disturbance degraded state following the end of the event and before the restoration is initiated $\left(t \in\left[t_{e e}, t_{o r}\right]\right.$ and $t \in\left[t_{e e}, t_{i r}\right]$ for the operational and infrastructure resilience respectively), and

\footnotetext{
${ }^{2} \mathrm{~A}$ linear approximation for the transitions between the resilience levels/states during the event is used for demonstration purposes; however, in reality, these state transitions can have any shape depending on the network constraints and prevailing conditions, and the impact of the event that hits the network
} 
- $\quad$ Phase III, restorative state $\left(t \in\left[t_{o r}, T_{o r}\right]\right.$ and $t \in\left[t_{i r}, T_{i r}\right]$ for the operational and infrastructure recovery respectively).

The features that a resilient power system must possess for responding effectively during these phases are given in [8].

During Phase $I$, the resilience level drops from the predisturbance resilience $R_{0 o}$ and $R_{0 i}$ to the post-disturbance infrastructure resilience, $R_{p d i}$, and to the post-disturbance operational resilience, $R_{p d o}$, respectively. It has to be noted that $R_{p d o}$ may be lower or higher than $R_{p d i}$, depending on the system and on the severity of the event hitting the network. It is thus system- and event-specific.

In Phase II, the system resides at the post-disturbance degraded operational and infrastructure state (i.e., $R_{p d o}$ and $R_{p d i}$ respectively) for some time before the operational and infrastructure restoration is initiated at $t_{o r}$ and $t_{i r}$ respectively. The duration of this period can differ for the infrastructure and operational resilience depending on the resilience solutions in place, e.g. smart operational solutions may initiate the load restoration (i.e., operational resilience) faster than the infrastructure recovery, which is a desirable situation.

Similarly, Phase III can be divided in two sub-phases: the operational recovery, $t \in\left[t_{o r}, T_{o r}\right]$, and the infrastructure recovery, $t \in\left[t_{i r}, T_{i r}\right]$. As will be illustrated in the case study application and as it is in most of the cases in reality, the operational resilience is restored faster than the infrastructure resilience. For example, the customers might be fully reconnected before the affected infrastructure components (e.g., collapsed transmission lines or towers, flooded substations, etc.) are fully restored. This is an additional reason why it is critical to make the distinction between operational and infrastructure resilience and to evaluate these concepts independently and using different indicators for capturing their distinct characteristics.

\section{B. The ФАЕП quantitative resilience metric framework}

In order to quantify the resilience of a power system, it is critical to define a set of metrics capable of capturing its performance during the different phases of the resilience trapezoid. Table I presents the key resilience metrics proposed here to characterize the resilience trapezoid, specifically for how fast $(\Phi)$ and how low $(\Lambda)$ resilience drops in Phase I, how extensive $(E)$ is the post-event degraded state (Phase II) and how promptly $(\Pi)$ the network recovers to its pre-event resilient state (Phase III), considering both operational and infrastructure resilience in each phase. This set of four metrics is defined in this work as the $\Phi \Lambda E \Pi$ resilience metric system (“ФАEП" is pronounced like " $F L E P$ ").

As seen in Table II, the $\Phi$-metric in Phase $I$ is evaluated by estimating the slope of the resilience degradation during the event (where $t_{e e}-t_{o e}$ is the duration of the windstorm, i.e., $t_{\text {windstorm }}$ ), while the $\Lambda$-metric is defined by the resilience degradation level, i.e., $R_{0 i}-R_{p d i}$ and $R_{0 o}-R_{p d o}$ for the infrastructure and operational resilience respectively. The $E$ metric showing the time that the network remains in the postdisturbance degraded state (Phase II) is given by $t_{o r}-t_{e e}$ and $t_{i r^{-}}$ $t_{e e}$ for the operational and infrastructure resilience respectively. The I-metric in Phase III is defined by the slopes of the operational and infrastructure recovery curves,
TABLE I

THE $Ф$ $П \Pi$ RESILIENCE METRIC SYSTEM

\begin{tabular}{c|c|c|c}
\hline Phase & State & Resilience metric & Symbol \\
\hline I & $\begin{array}{c}\text { Disturbance } \\
\text { progress }\end{array}$ & $\begin{array}{c}\text { How fast resilience drops? } \\
\text { How low resilience drops? }\end{array}$ & $\begin{array}{c}\Phi \\
\Lambda\end{array}$ \\
\hline II & $\begin{array}{c}\text { Post-disturbance } \\
\text { degraded }\end{array}$ & $\begin{array}{c}\text { How extensive is the post- } \\
\text { disturbance degraded state? }\end{array}$ & $E$ \\
\hline III & Restorative & $\begin{array}{c}\text { How promptly does the network } \\
\text { recover? }\end{array}$ & $\Pi$ \\
\hline
\end{tabular}

TABLE II

MATHEMATICAL FORMULATION OF RESILIENCE METRICS

\begin{tabular}{|c|c|c|c|c|}
\hline \multirow{2}{*}{ Metric } & \multicolumn{2}{|c|}{ Mathematical Expression } & \multicolumn{2}{|c|}{ Measuring Unit } \\
\hline & Operational & Infrastructure & Operational & Infrastructure \\
\hline$\Phi$ & $\frac{R_{p d o}-R_{O o}}{t_{e e}-t_{o e}}$ & $\frac{R_{p d i}-R_{O i}}{t_{e e}-t_{o e}}$ & MW/hours & $\begin{array}{l}\text { Number of } \\
\text { lines } \\
\text { tripped/hours }\end{array}$ \\
\hline$\Lambda$ & $R_{O o}-R_{p d o}$ & $R_{O i}-R_{p d i}$ & MW & $\begin{array}{l}\text { Number of } \\
\text { lines tripped }\end{array}$ \\
\hline$E$ & $t_{o r}-t_{e e}$ & $t_{i r}-t_{e e}$ & Hours & Hours \\
\hline$\Pi$ & $\frac{R_{O o}-R_{p d o}}{T_{o r}-t_{o r}}$ & $\frac{R_{0 i}-R_{p d i}}{T_{i r}-t_{i r}}$ & MW/hours & $\begin{array}{c}\text { Number of } \\
\text { lines } \\
\text { restored/hours }\end{array}$ \\
\hline Area & $\int_{t_{o e}}^{T_{o r}} R_{o p}(t) d t$ & $\int_{t_{o e}}^{T_{i r}} R_{i}(t) d t$ & MW $\times$ hours & $\begin{array}{l}\text { (Number of } \\
\text { lines in } \\
\text { service) } \times \text { hours }\end{array}$ \\
\hline
\end{tabular}

TABLE III

MATHEMATICAL EXPRESSION OF THE LINEARIZED TRAPEZOID AREAS

\begin{tabular}{c|c|c}
\hline \multirow{2}{*}{$\begin{array}{c}\text { Trapezoid } \\
\text { Area }\end{array}$} & \multicolumn{2}{|c}{ Mathematical Expression } \\
\cline { 2 - 3 } Area $_{I}$ & $\frac{\Lambda_{\text {operational }} \times t_{\text {windstorm }}}{2}$ & $\frac{\Lambda_{\text {infrastructure }} \times t_{\text {windstorm }}}{2}$ \\
\hline Area $_{I I}$ & $\Lambda_{\text {operational }} \times E_{\text {operational }}$ & $\Lambda_{\text {infrastructure }} \times E_{\text {infrastructure }}$ \\
\hline Area $_{I I I}$ & $\frac{\Lambda_{\text {operational }} \times\left(T_{\text {or }}-t_{\text {or }}\right)}{2}$ & $\frac{\Lambda_{\text {infrastructure }} \times\left(T_{\text {ir }}-t_{\text {ir }}\right)}{2}$ \\
\hline
\end{tabular}

which consider both the original pre-event ${ }^{3}$ resilience level and the time required for reaching this resilience level.

Further to the $\Phi \Lambda E \Pi$ resilience metrics, an additional metric is used, i.e., the area of the trapezoid. As shown in Table II, the area metric would be expressed as the integral of the trapezoid for the duration of the event, again taking into account the operational and infrastructure resilience. Considering piecewise linearity for the different phases of the resilience trapezoid of Fig. 2 (which results in two right triangles for Phases $I$ and III and one rectangular for Phase II), the areas corresponding to the three phases of the resilience trapezoid (I, II and $I I I)$ can be approximated as shown in Table III. The total area of the operational and infrastructure resilience trapezoid is given by the sum of Area $_{I}$, Area $_{I I}$ and Area ${ }_{I I I}$. It can be seen that the area metric is dependent on the $\Phi \Lambda E \Pi$ metrics (mainly on the $\Lambda$ and $E$ metrics) defined and mathematically expressed in Tables I and II.

\footnotetext{
${ }^{3}$ In principle, a "post-restoration" resilience level could also be defined which might differ from the original pre-disturbance level. For simplicity, here we do not explicitly deal with this aspect which can be case specific, but the proposed framework is capable to address it.
} 
Different resilience indicators can be used to express and quantify these resilience metrics. It is critical to use indicators that capture both the prevailing operational and infrastructure resilience during the event. In this study where the focus is on assessing the resilience of transmission networks to extreme weather events, the following indicators are used ${ }^{4}$ :

- the amount of generation capacity (MW) and load demand (MW) that are connected during the event are used as indicators for the operational resilience; and

- the number of online transmission lines is used as an indicator for the infrastructure resilience.

Based on these indicators, the measuring units of the resilience metrics are also defined in Table II.

Hence, a set of five resilience metrics is proposed in this work for effectively and systematically quantifying the resilience of a power system subject to an external shock. This quantitative resilience metric framework includes the four independent $\Phi \Lambda E \Pi$ metrics, and the area metric which is dependent on the $\Phi \Lambda E \Pi$ metrics. The resilience assessment of a power system using this framework would enable the indepth understanding of the resilience level of the power system and drive the utilization of targeted resilience enhancement strategies for improving the resilience of the system if and during the specific phase of the resilience trapezoid it is deemed inadequate.

\section{FRAGILITY ModelLING AND RESILIENCE ASSESSMENT OF A TRANSMISSION NETWORK TO EXTREME WEATHER}

In order to model the fragility of a transmission network to a weather event, the concept of fragility curves is used here, which express the failure probability of a component as a function of the weather parameter, e.g. wind speed. By mapping the weather profile at each simulation step to these curves, the weather- and time-dependent failure probability of a transmission corridor can be obtained. A generic fragility curve is shown in Fig. 3, whose shape varies and depends on the application, but it can be expressed as follows:

$$
P\left(w_{i}\right)= \begin{cases}0, & \text { if } w<w_{\text {critical }} \\ P(w), & \text { if } w_{\text {critical }} \leq w<w_{\text {collapse }} \\ 1, & \text { if } w \geq w_{\text {collapse }}\end{cases}
$$

where $P\left(w_{i}\right)$ is the failure probability of the component as a function of the weather parameter $(w)$ at simulation step $i$, $w_{\text {critical }}$ is the value of the weather parameter at which the failure probability picks-up and $w_{\text {collapse }}$ represents the value of the weather parameter at which the failure of the component is certain, i.e., $P_{i}(w)=1$. The transmission corridor will trip at the next simulation step if $P_{i}(w)>r$, where $r$ is a uniformly distributed random number $(r \sim U(0,1))$. If $P_{i}(w)<r$, the corridor will not trip. The restoration time is randomly generated if a transmission corridor outage occurs, which is also weatherdependent as will be discussed later.

The weather- and time-dependent operational modes of the corridors are then fed to a Sequential Monte Carlo simulation-

\footnotetext{
${ }^{4}$ Other similar indicators could of course also be used, depending on the specific study and objectives of the analysis.
}

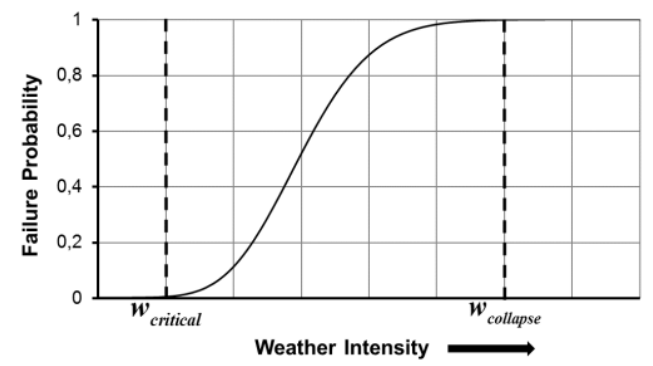

Fig. 3. A generic fragility curve showing the failure probability of a component as a function of the weather intensity

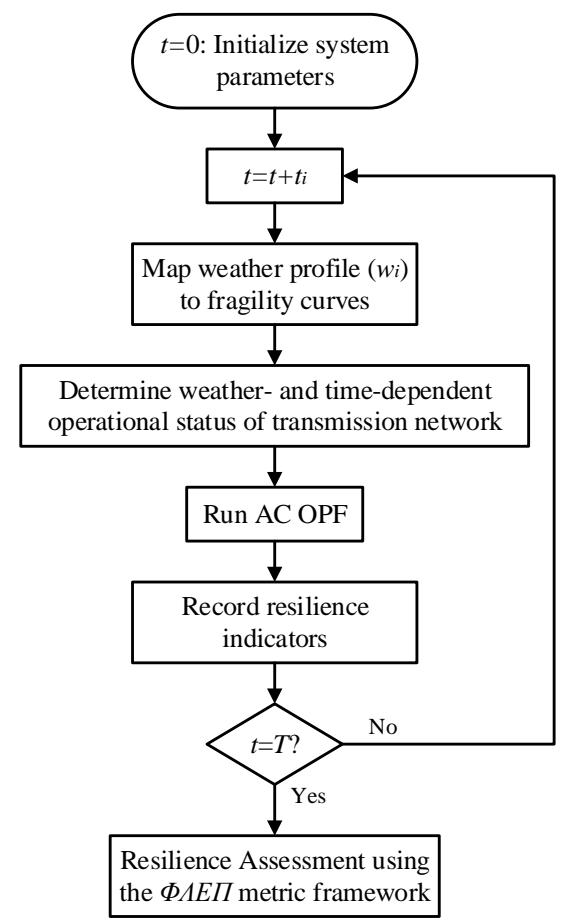

Fig. 4. System approach for assessing the resilience of transmission networks to weather events

based engine to capture the multi-temporal and multi-spatial impact of the weather front as it moves across the transmission network. Fig. 4 shows the generic approach followed for obtaining the weather-dependent resilience indicators and applying the $\Phi \Lambda E \Pi$ resilience metric framework. It is worth mentioning here that even though $\mathrm{AC}$ OPF is considered an appropriate dispatch tool for this specific application where the focus is on developing and illustrating a complete resilience quantification framework, it does not consider other important relevant issues, such as, e.g., the ramping rates of the generators. However, the proposed tool offers the capability and flexibility to include such constraints for modelling additional dimensions of the problem.

In particular, regarding the calculation of the operational resilience indicators, the amount of the generation capacity connected at each simulation step is taken as the capacity that can be provided given the prevailing transmission network topology, connectivity and constraints during the event. It has to be clarified that this indicator is different from the generation output at each simulation step. Also, for the purposes of the AC OPF implementation, the load shedding that might occur at each simulation step (which enables the calculation of the connected load) is calculated using an objective function for the minimization of the load shedding, which considers the power flows and network constraints, 
such as generation and transmission capacities and voltage operational limits. These resulting non-linearities are considered in the calculation of the operational indicators. Finally, the infrastructure resilience indicator is calculated by recording the status of the transmission lines at each simulation step.

\section{CASE StUdy APPliCATION}

The proposed approach is demonstrated in this Section through various case studies on the GB test system to illustrate different aspects and the metrics of the resilience trapezoid.

\section{A. Test network and simulation data}

The test version of the GB transmission network of Fig. 5 is used here, for which further details can be found in [18, 19]. The node demands (derived from [18] and [20]) are fluctuating and are time-varying in the simulations, but it is assumed that there is no impact of the weather event on the demand profiles. The generation is then dispatched at each simulation step through the AC OPF to meet this demand, taking into account the network constraints.

As the focus is on assessing the impact of windstorms on the transmission network resilience, and in particular on transmission lines and towers, the wind fragility curves of these components are required. Fig. 6 shows the transmission tower and lines' wind fragility curves used in this application (for the base and robust case scenarios) [16, 21].

The duration of the windstorm is assumed to be $24 \mathrm{~h}$, with an hourly time resolution. It is also assumed that the severe windstorm hits the network at $50 \mathrm{~h}$ from the beginning of the simulation and that all the components are online before the event occurs. The hourly wind profiles along the transmission corridors of the test network in GB are obtained using MERRA re-analysis, as discussed in [19], which provides weather data based on satellite observations. As high wind speeds that can threaten the transmission network resilience are rare in the MERRA wind profiles, these are scaled-up by using appropriate multiplication factors for the entire wind profile. In particular, three windstorm profiles are generated, with maximum wind speeds $\left(w_{\max }\right)$ equal to $40 \mathrm{~m} / \mathrm{s}, 50 \mathrm{~m} / \mathrm{s}$ and $60 \mathrm{~m} / \mathrm{s}$ respectively (referred as $\mathrm{WS}_{40 \mathrm{~m} / \mathrm{s}}, \mathrm{WS}_{50 \mathrm{~m} / \mathrm{s}}$ and $\mathrm{WS}_{60 \mathrm{~m} / \mathrm{s}}$ in the rest of the paper), which according to the Beaufort wind force scale by the Met Office, UK [22] represent hurricanes. This approach is followed in order to model the worst conditions that the test network might experience.

A Mean Time To Repair $\left(M T T R_{\text {normal }}\right)$ of $10 \mathrm{~h}$ and $50 \mathrm{~h}$ is assumed for the transmission lines and towers respectively. Random times to repair $(T T R)$ based on the $M T T R_{\text {normal }}$ of the transmission lines and towers are then generated once a fault/collapse of a component occurs during the windstorm, which follow an exponential distribution. As aforementioned, it is considered that no restoration takes place during the extreme event mainly due to safety reasons, so the repair crews are dispatched (and thus the TTR starts) at the end of the event. The approach followed for modelling the increasing time to repair the damaged components for higher wind speeds is given in [19], which basically uses multiplication factors for higher wind speeds to model the higher damage caused by the windstorm and the increasing difficulty in approaching the affected areas and restoring the faulted components.

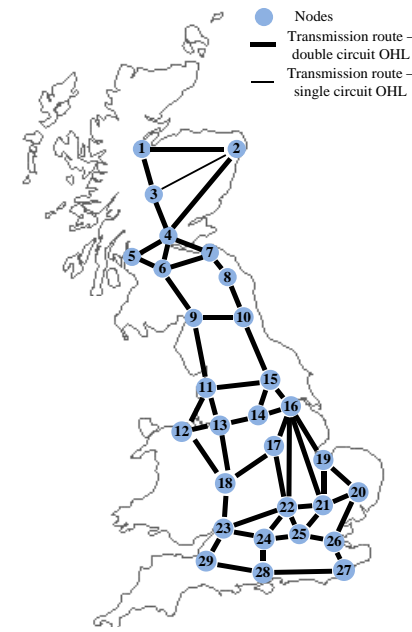

Fig. 5. The test version of Great Britain transmission network [18]

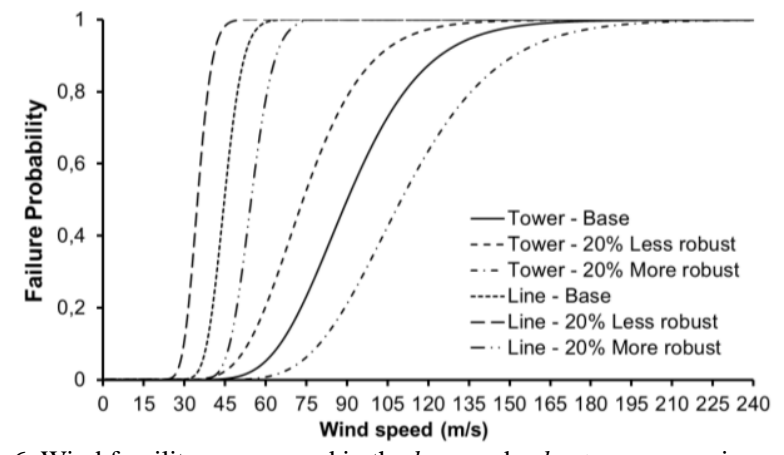

Fig. 6. Wind fragility curves used in the base and robust case scenario

\section{B. Resilience assessment to a grid-scale windstorm}

Different operational and infrastructure aspects can affect the resilience performance of a power system subject to extreme weather. Within this context, several case studies have been systematically developed to evaluate the impact of these aspects on the resilience of the test network, as this is quantified using and to test the suitability of the proposed $\Phi \Lambda E \Pi$-based resilience metric framework. In particular, the following illustrative scenarios are considered:

- Base: The resilience trapezoid is modelled with the data of Section IV-A and for $\mathrm{WS}_{40 \mathrm{~m} / \mathrm{s}}, \mathrm{WS}_{50 \mathrm{~m} / \mathrm{s}}$ and $\mathrm{WS}_{60 \mathrm{~m} / \mathrm{s} \text {. }}$

- Robust: In this case scenario, the transmission lines and towers are made $20 \%$ more and less robust to the windstorm, by adjusting the fragility curves (see Fig. 6).

- Response: As in the robust case scenario, the responsiveness to the weather event is made $20 \%$ better and worse, by decreasing and increasing the $M T T R_{\text {normal }}$ assumed for the transmission lines and towers by $20 \%$.

- Resources: A critical aspect is the amount of resources, i.e., repair crews (RCs), for responding quickly and effectively to the collapsed components during weather events. Hence, in this case scenario, the effect of unlimited number of repair crews, 5, 10 and 15 is evaluated. It is considered that one repair crew is needed for each damaged transmission corridor, which introduces a limitation on the number of corridors that can be repaired at the same time.

It is also assumed that no restoration takes place during the event (i.e., $t \in\left[t_{o e}, t_{e e}\right]$ ). These case scenarios have been based 


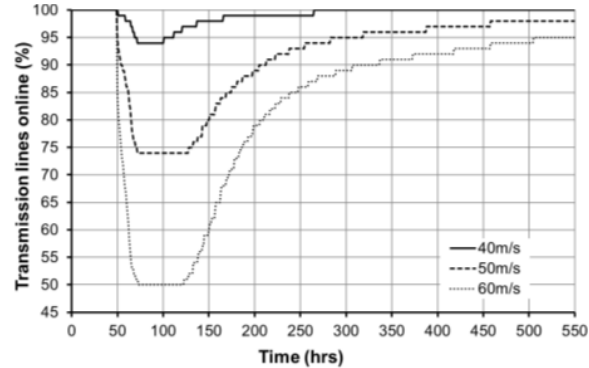

a) Transmission lines online (\%)

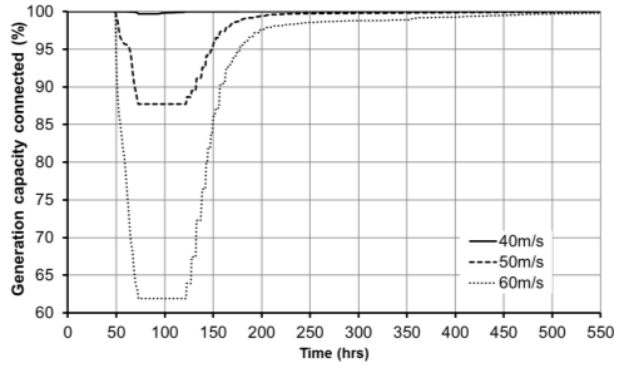

b) Generation capacity connected (\%)

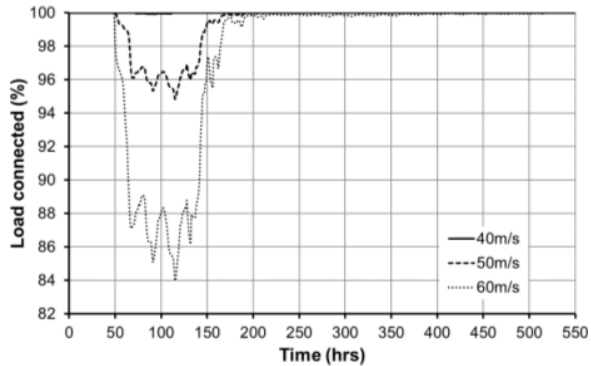

c) Load connected (\%)

Fig. 7. Resilience indicators as a function of time for the base case scenario

on engineering judgments and discussions with National Grid, the GB transmission system operator. It has to be noted here that the case scenarios robust, response and resources are applied only for $\mathrm{WS}_{50 \mathrm{~m} / \mathrm{s}}$; similar conclusions can be obtained by applying these scenarios for the other windstorms.

\section{1) Obtaining the time-dependent resilience indicators}

Fig. 7 shows the time-dependent resilience indicators for the base scenario and the three windstorms modelled, i.e.,

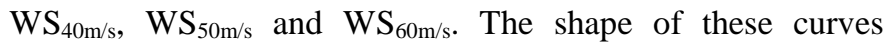
clearly recall the resilience trapezoid of Fig. 2, enabling its proper modeling and quantification; that is, the three phases can be clearly distinguished: disturbance progress, post-event degraded state and restoration phase. The duration of Phase I is known and equal to the duration of the extreme event (i.e., 24h in this work), while the duration of Phases II and III would be the output of the simulation engine and, as demonstrated later, would depend on the emergency and preparedness plans in place by the electric utility (i.e., the response and RCs case studies considered in this work). In general, Phase II starts at the end of the event $\left(t_{e e}\right)$ and ends when the operational and infrastructure resilience start recovering ( $t_{o r}$ and $t_{i r}$ respectively), e.g. when some demand or a damage component is restored. Similarly, Phase III starts at $t_{o r}$ and $t_{i r}$ and ends at $T_{o r}$ and $T_{i r}$ respectively, i.e., when the operational and infrastructure resilience fully recover.

Further, as illustrated in Fig. 2, it can be observed that the operational resilience degradation $\left(R_{p d o}\right)$ as obtained by the operational resilience indicators in Figs. 7.b and 7.c is significantly lower than the infrastructure resilience degradation $\left(R_{p d i}\right)$ in Fig. 7.a. It can also be seen that the operational indicators of generation and load connected recover much faster than the infrastructure indicator of the transmission lines online. This proves the importance and the need for distinguishing the concepts of infrastructure and operational resilience, as well as the use of different resilience indicators for their quantification.

\section{2) Application of the $Ф$}

Based on the above resilience indicators, it is now possible to quantify the system resilience via the proposed metrics.

The first metric to evaluate is the $\Phi$-metric, i.e., how fast the operational and infrastructure resilience levels degrade. Table IV shows the $\Phi$-metric of the resilience degradation curves of Phase $I$ of the resilience trapezoid for the infrastructure and operational resilience indicators. These slopes are negative, as resilience degrades. It can be clearly seen that the degradation slopes increase for higher wind
TABLE IV

THE $\Phi$-METRIC For THE BASE CASE SCENARIO

\begin{tabular}{|c|c|c|c|}
\hline \multirow{2}{*}{ Event } & \multicolumn{3}{|c|}{ Resilience Indicator } \\
\hline & Trans. lines & Gen. Connected & Load Connected \\
\hline $\mathrm{WS}_{40 \mathrm{~m} / \mathrm{s}}$ & -0.2500 & -0.0125 & -0.0024 \\
\hline $\mathrm{WS}_{50 \mathrm{~m} / \mathrm{s}}$ & -1.0833 & -0.521 & -0.249 \\
\hline $\mathrm{WS}_{60 \mathrm{~m} / \mathrm{s}}$ & -2.0833 & -1.5876 & -0.6668 \\
\hline
\end{tabular}

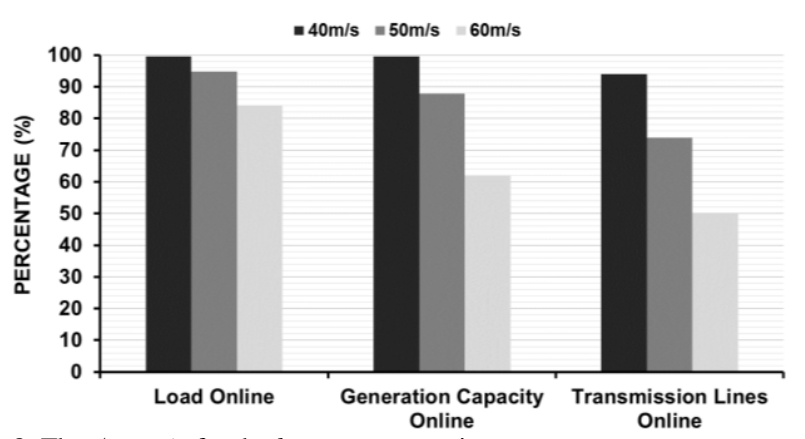

Fig. 8. The 1-metric for the base case scenario

TABLE V

THE $\Phi$-METRIC For THE ROBUST CASE SCENARIO AND WS $\mathrm{W}_{50 \mathrm{M} / \mathrm{s}}$

\begin{tabular}{c|ccc}
\hline Event & Trans. lines & $\begin{array}{c}\text { Resilience Indicator } \\
\text { Gen. Connected }\end{array}$ & Load Connected \\
\hline $\begin{array}{c}20 \% \text { Less } \\
\text { Robust }\end{array}$ & -2.2083 & -1.8083 & -0.7132 \\
Base & -1.0833 & -0.521 & -0.249 \\
20\% More & -0.2500 & -0.0121 & -0.0117 \\
Robust & &
\end{tabular}

speeds for all the resilience indicators. Further, the highest slopes occur for the infrastructure indicator of transmission lines online, followed by the operational resilience indicators.

The second metric to evaluate within the $\Phi \Lambda E \Pi$ framework is the 1-metric, i.e., how low resilience drops in Phase I. Fig. 8 shows this metric for the base case scenario, as expressed using the infrastructure and operational resilience indicators. As expected, the resilience degradation increases for higher wind speeds. Also, as shown in Fig. 7 as well, the highest impact occurs on the infrastructure resilience for all the windstorm intensities evaluated here.

Table V shows the $\Phi$-metric and Fig. 9 the 1 -metric for the robust case scenario, which is the scenario with the largest effect on these metrics, for $\mathrm{WS}_{50 \mathrm{~m} / \mathrm{s}}$. It can be seen that boosting (reducing) the robustness of the transmission corridors improves (worsens) the slope and level of resilience degradation in Phase I. 


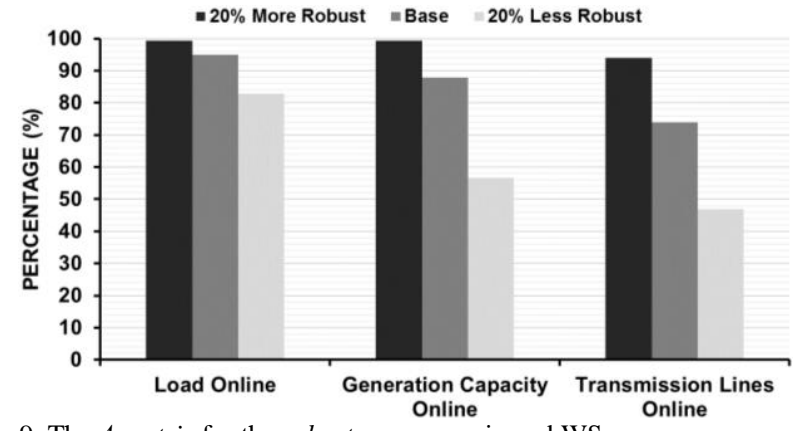

Fig. 9. The 1 -metric for the robust case scenario and $\mathrm{WS}_{50 \mathrm{~m} / \mathrm{s}}$.

TABLE VI

The E-METRIC For The RESPONSE CASE SCENARIO AND WS $\mathrm{W}_{50 \mathrm{M} / \mathrm{s}}$

\begin{tabular}{|c|c|c|c|}
\hline \multirow[b]{2}{*}{ Case study } & \multicolumn{3}{|c|}{ Duration of post-event degraded state (hours) } \\
\hline & $\begin{array}{c}\text { Transmission } \\
\text { lines }\end{array}$ & $\begin{array}{c}\text { Generation } \\
\text { Capacity }\end{array}$ & Load \\
\hline 20\%MoreResponse & 44 & 47 & 48 \\
\hline Base & 53 & 54 & 57 \\
\hline $20 \%$ LessResponse & 76 & 80 & 83 \\
\hline
\end{tabular}

TABLE VII

THE $\Pi$-METRIC IN PHASE III For THE RESPONSE AND RESOURCES CASE SCENARIOS AND WS $\mathrm{W}_{50 \mathrm{~m} / \mathrm{s}}$

\begin{tabular}{c|ccc}
\hline \multirow{2}{*}{ Case Study } & \multicolumn{3}{|c}{ Resilience indicator } \\
& Trans. lines & Gen. Connected & Load Connected \\
\hline 5 RCs & 0.0128 & 0.0060 & 0.0026 \\
$10 \mathrm{RCs}$ & 0.0137 & 0.0069 & 0.0039 \\
$15 \mathrm{RCs}$ & 0.019 & 0.0148 & 0.0076 \\
20\%LessResponse & 0.0455 & 0.0266 & 0.0111 \\
Base & 0.0578 & 0.0330 & 0.0724 \\
20\%MoreResponse & 0.0925 & 0.0354 & 0.0925 \\
\hline
\end{tabular}

Table VI demonstrates the E-metric, i.e., how long is the duration of the post-disturbance degraded state (Phase II), for the base and response case scenarios for $\mathrm{WS}_{50 \mathrm{~m} / \mathrm{s}}$. It is shown that improving (resp. worsening) the responsiveness to the event results in lower (resp. higher) durations of the postevent degraded state for all the resilience indicators. This scenario does not have an impact on the $\Phi$ and $\Lambda$ metrics of Phase I. Also, the robust and resources scenarios do not have a great effect on the E-metric.

The last metric to evaluate within the $\Phi \Lambda E \Pi$ framework is the $\Pi$-metric, i.e., how fast the operational and infrastructure resilience levels recover in Phase III. For this purpose, Table VII shows the recovery slopes of the response and resources scenarios for $\mathrm{WS}_{50 \mathrm{~m} / \mathrm{s}}$ (the robust scenario does not affect this metric). As can be seen, different recovery slopes correspond to the infrastructure and operational indicators. Further, it is apparent that the limitation of the repair crews has the greatest effect on the restoration capability of the restoration slopes of both infrastructure and operational resilience. Also, the higher the number of repair crews, the higher the number of transmission corridors that can be fixed at the same time and hence the higher the restoration slopes (i.e., П-metric) of all the resilience indicators.

\section{3) Calculation of the area metric}

Based on the resilience metrics estimated within the $\Phi \Lambda E \Pi$ resilience framework in the previous subsection, the area metric is next estimated.

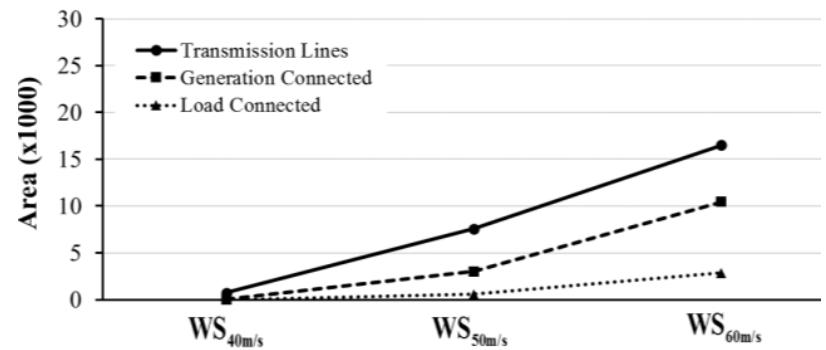

Fig. 10. The area metric for $\mathrm{WS}_{40 \mathrm{~m} / \mathrm{s}}, \mathrm{WS}_{50 \mathrm{~m} / \mathrm{s}}$ and $\mathrm{WS}_{60 \mathrm{~m} / \mathrm{s}}$

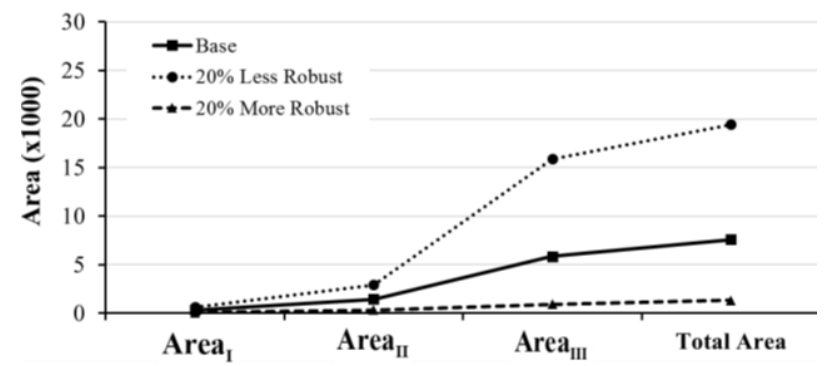

Fig. 11. The area metric of the indicator transmission lines online for $\mathrm{WS}_{50 \mathrm{~m} / \mathrm{s}}$ for the robust case scenario and the phases of the trapezoid

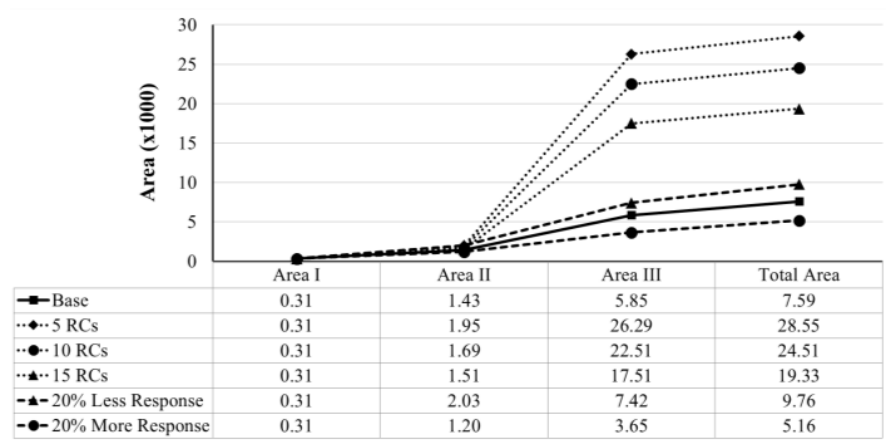

Fig. 12. Area metric of the indicator transmission lines online for $\mathrm{WS}_{50 \mathrm{~m} / \mathrm{s}}$. Results shown for the case scenarios base, response and resources (RCs).

Fig. 10 shows the total area metric for $\mathrm{WS}_{40 \mathrm{~m} / \mathrm{s}}, \mathrm{WS}_{50 \mathrm{~m} / \mathrm{s}}$ and $\mathrm{WS}_{60 \mathrm{~m} / \mathrm{s}}$ for the different resilience indicators. The larger is the area, the larger is the overall impact by the windstorm. Therefore, as aforementioned, the highest impact is observed on the infrastructure resilience. Further, the area metric increases significantly for higher wind speeds.

Figs. 11 and 12 show the area metrics for the different phases of the resilience trapezoid using the indicator of transmission lines online for $\mathrm{WS}_{50 \mathrm{~m} / \mathrm{s}}$ and for the robust, response and resources ( $\mathrm{RCs}$ ) case scenarios respectively. Similar trends are observed for the operational indicators. It can be seen that the robust case scenarios (Fig. 11) result in a large difference in Area $_{I I}$ due to the large difference in the resilience degradation. However, this variance is lower in the case scenarios of response and RCs (Fig. 12) due to the smaller difference in the duration of Phase II in these scenarios. Further, Fig. 12 shows that Area $_{I}$ is equal for the response and RCs scenarios, as the resilience degradation is the same for these scenarios; only the post-event restoration time is influenced. It can also be seen that Area $_{I I I}$ is

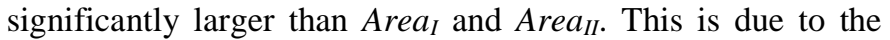
much longer duration of Phase III compared to Phases I and II. Further, this is because the value of the Total Area is close to the value of Area $a_{I I I}$. Also, in Fig. 12 it is shown again that the limitation of repair crews has a much higher effect on Area $_{I I I}$ than the response case scenarios. 


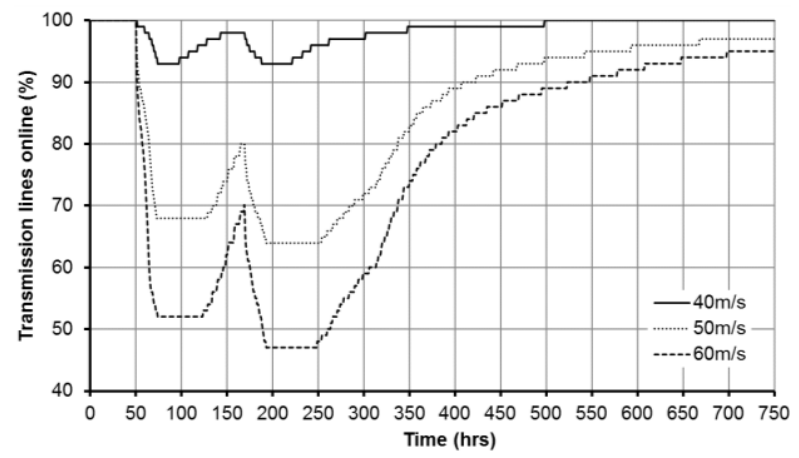

Fig. 13. Transmission lines online (\%) when the test system is subject to two, successive windstorms, for the base scenario (5 days between windstorms)

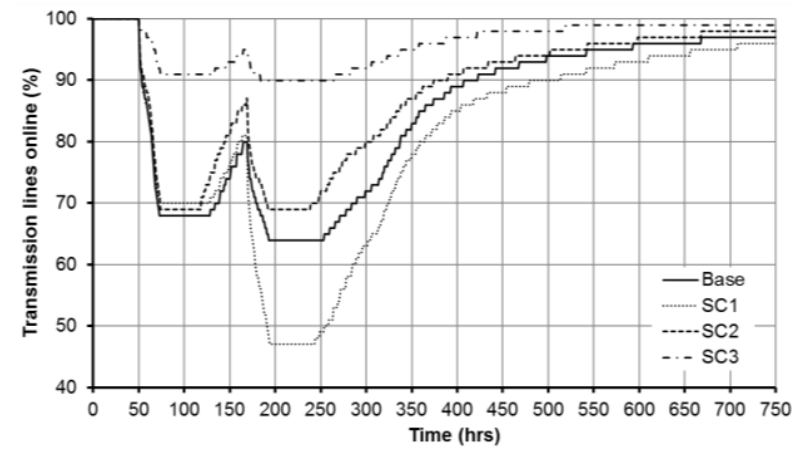

Fig. 14. Transmission lines online (\%) when the test system is subject to two, successive windstorms, for different scenarios

\section{Resilience assessment to grid-scale multiple windstorms}

The resilience of the test network when it is subject to two successive events is evaluated next. For brevity, only the timedependent infrastructure resilience indicator of transmission lines online is provided here (similar trends hold for the operational indicators). The complete $\Phi \Lambda E \Pi$-based resilience metric framework can be applied following the same approach as in the single weather event in the previous subsections.

In particular, for the base scenario, it is first assumed that the test network is hit by two windstorms of the same intensity $\left(\mathrm{WS}_{40 \mathrm{~m} / \mathrm{s}}, \mathrm{WS}_{50 \mathrm{~m} / \mathrm{s}}\right.$ and $\left.\mathrm{WS}_{60 \mathrm{~m} / \mathrm{s}}\right)$ with a five-day interval between the two events, i.e., the first event is applied at $50 \mathrm{~h}$ and the second event at $170 \mathrm{~h}$, both with a $24 \mathrm{~h}$ duration.

Fig. 13 shows the online transmission lines (\%) as a function of time. Likewise the single event, the lowest impact and fastest restoration is observed for $\mathrm{WS}_{40 \mathrm{~m} / \mathrm{s}}$, which enables recovery so that the system can effectively deal with the second event. In the case of $\mathrm{WS}_{50 \mathrm{~m} / \mathrm{s}}$ and $\mathrm{WS}_{60 \mathrm{~m} / \mathrm{s}}$, due to the higher impact and the partial restoration of the infrastructure resilience before the occurrence of the second event, further infrastructure resilience degradation is observed, i.e., the percentage of lines tripped increases after the second event.

Fig. 14 shows a comparison of different scenarios, namely base for $\mathrm{WS}_{50 \mathrm{~m} / \mathrm{s}}$, different intensity of the windstorms (SC1, the first windstorm that is applied is $\mathrm{WS}_{50 \mathrm{~m} / \mathrm{s}}$ and the second $\mathrm{WS}_{60 \mathrm{~m} / \mathrm{s}}$ ) and improving the responsiveness and robustness by $20 \%$ (SC2 and SC3 respectively) for $\mathrm{WS}_{50 \mathrm{~m} / \mathrm{s}}$. The time period between the events is $120 \mathrm{~h}$. In $S C 1$, the higher intensity of the second event causes a much higher infrastructure resilience degradation, even though some resilience recovery takes place before it occurs. The more effective and faster response in SC2 enables higher and quicker recovery following the first event, which results in a lower resilience decrease following the second event, compared to the base scenario. The lowest resilience degradation is for $S C 3$, during both windstorms.

\section{Smart operational measure ("defensive islanding") and} resilience assessment to region-scale windstorm

In the previous sections, the resilience of the test network to a wide grid-scale windstorm has been evaluated, which is considered to be the worst-case scenario in terms of the area hit by the weather event. In this section, a region-scale windstorm is modelled. Particularly, the windstorm hits the North and Central-East region of the test network, and more specifically the transmission corridors connecting nodes 1-10 and 14-17 (see Fig. 5). According to historical data [20, 23], this GB region is being hit hard and quite frequently by severe winds. Further, in order to evaluate the effect of the failure propagation that might be caused by the regional windstorm to the rest of the network, the cascading mechanism is considered and modelled in this study, as illustrated in [19].

In [19], a defensive islanding (DI) scheme based on a severity risk index is developed and thoroughly presented, in order to determine the effect of "smart" operational measures to the resilience enhancement of a power system to extreme weather events. In this work, this DI scheme is used as a case study in order to demonstrate the capability of the resilience metric framework to quantify the contribution of such "smart" operational strategies.

Only the cascading caused by thermal overloads is considered and the lines that are tripped by the protection equipment due to thermal overloads (these lines are not damaged by the windstorm) are reconnected the next hour. Lines reconnection is important as it increases the options for defensive islanding (possible formations of islands in the next simulation step) when its application is decided and reduces the possibility of congestion. It is considered that the time period of one hour is enough in order to reconnect the lines after their tripping by the protection due to thermal overloads. Transient phenomena during reconnection of the lines are not examined. In addition, it is noted that the reconnections are applied in a controlled way and therefore the transient phenomena that could lead to instability are assumed unlikely.

Defensive islanding isolates the region which is hit by extreme weather to prevent the cascading events to be spread to the rest of the network. The reconnection of islands after the application of $D I$ is discussed in [19]. In order to model highly stressed conditions which would enable the effective demonstration of the contribution of $D I$ to the resilience of the test network, the hourly load demand is increased by $20 \%$ here and the resilience of the system is tested using maximum wind speeds higher than $40 \mathrm{~m} / \mathrm{s}$ [19].

$D I$ is not able to prevent the direct damage of a line due to a windstorm. It is applied to prevent cascading events that are triggered due to line damages by the extreme weather. Since the tripped lines by the protection due to thermal overloads are reconnected in the next hour, the number of damaged lines due to windstorm will be the same with or without $D I$ at the end of Phase I. Therefore, the operational measure DI is applied during Phase $I$ and is able to enhance the resilience of the system only in Phase I of Fig. 2, as the overloaded lines are reconnected in the next hour. Hence, for demonstrating clearer the contribution of DI, Fig. 15 shows the transmission lines and load online (\%) with and without DI only for Phase I of 


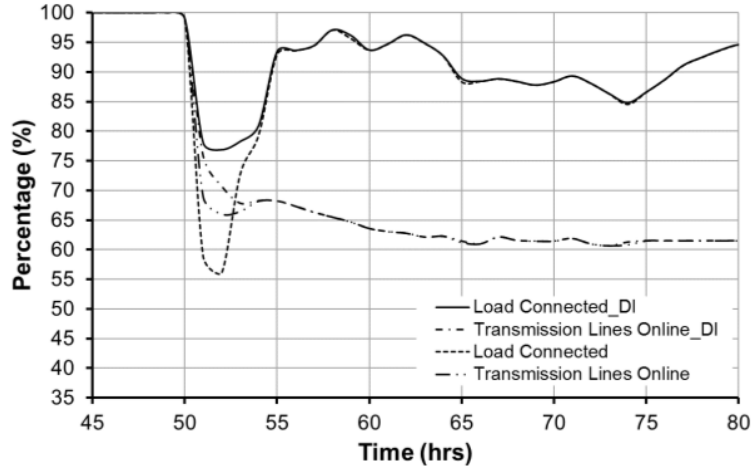

Fig. 15. Transmission lines and load online during the event (Phase I only) with/without the application of defensive islanding for a grid-scale windstorm

the resilience trapezoid (time of windstorm $=50 \mathrm{~h}$, duration of windstorm $=24 \mathrm{~h}$ ). During the first hours of the extreme weather event the resilience of the system is significantly improved with $D I$, as it can be observed that the resilience indicators with $D I$ are higher than the ones without $D I$. This is because the larger number of tripped lines is usually observed in the first hours that the windstorm hits the system. This could thus possibly lead to cascading events, which deems the $D I$ as an effective solution for mitigating the effects of cascading outages. The next hours, due to the fact that several lines have already tripped, the number of failed lines is reduced. This makes the effect of $D I$ less critical.

Interestingly, the resilience curve in Fig. 15 is not monotonically descending in Phase $I$ as in previous sections where the cascading mechanism was not considered. Further, it can be seen that $D I$ affects the 1-metric, i.e., how low resilience drops at each hour of Phase I. It is also observed that after the first few hours that the windstorm hits the network, the connected load increases while the online transmission lines are further reduced. This is because in the South-East region of GB there are large urban centers (e.g. node 25, London) and their electrification can be interrupted due to the cascading events during the early stages of the event, but reconnected shortly after. It is noted that all the previous case studies can be applied in conjunction with $D I$ to investigate their combined effect on the resilience trapezoid.

\section{CONCLUSIONS}

A multi-phase resilience trapezoid has been discussed in this paper. This trapezoid has been methodically quantified using a novel resilience quantification framework based on the ФАEП (pronounced like "FLEP") resilience metrics, which, along with the area of the trapezoid, define a set of five resilience metrics specifically developed. By using different resilience indicators for quantifying these metrics, the proposed framework critically distinguishes the concepts of operational and infrastructure resilience, and enables the systematic modelling of the operational and infrastructure resilience performance of a power system subject to extreme

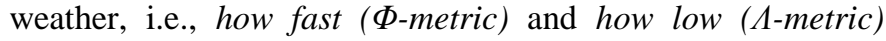
resilience drops, how extensive (E-metric) is the duration of the post-event degraded state and how promptly (II-metric) it reaches its pre-event state. Hence, the proposed approach enables the effective modelling of the time-dependent behavior of an infrastructure subject to extreme events.

The effect of different structural and operational resilience enhancement strategies can be quantified using the proposed framework. This can thus support adaptation policies, which is another critical time-dependent resilience feature.

The case study applications focused on assessing the resilience of a 29-bus test version of the GB transmission network to grid-scale single and multiple windstorms. The resilience of the test network to a region-scale windstorm, with the application of the smart operational strategy of defensive islanding, has also been assessed. The results clearly demonstrate the suitability, effectiveness and flexibility of the proposed framework and metrics set to quantify power systems resilience, evaluate the effect of different operational and infrastructure aspects on the behavior of a power system when it is exposed to extreme events, and assess the benefits from different resilience enhancement strategies.

Work in progress aims at illustrating how the proposed metrics framework can be used in a full cost/benefit analysis framework to support the quantitative decision making for fulfilling both resilience and cost goals.

\section{ACKNOWLEDGMENTS}

Mathaios Panteli and Pierluigi Mancarella would like to thank the UK EPSRC for the partial support to conduct this research through the "RESNET" (EP/I035757/1) and the "Disaster management and resilience in electric power systems" (EP/N034899/1) projects.

\section{REFERENCES}

[1] C. S. Holling, "Resilience and Stability of Ecological Systems," Annual Review of Ecology and Systematics, vol. 4, pp. 1-23, 1973.

[2] K. Tierney and M. Bruneau, "Conceptualizing and Measuring Resilience: A Key to Disaster Loss Reduction," TR News 250, MayJun. 2007.

[3] W. Neil Adger, "Social and ecological resilience: Are they related?," Progress in Human Geography, vol. 24, pp. 347-364, 2000.

[4] National Science and Technology Council, "Grand Challenges for Disaster Reduction," Subcommittee on Disaster Reduction, Washington DC, USA, 2005.

[5] A. Rose, "Economic resilience to natural and man-made disasters: Multidisciplinary origins and contextual dimensions," Environmental Hazards, vol. 7, no. 4, pp. 383-398, 2007.

[6] M. Lembani, A. Mohammed, A. Abdulwahab, A. Garba, H. d. Pinho, P. Delobelle, et al., "Health Systems Resilience: A Systems Analysis," Oct. 2014.

[7] M. Panteli and P. Mancarella, "Influence of Extreme Weather and Climate Change on the Resilience of Power Systems: Impacts and Possible Mitigation Strategies," Electr. Power Syst. Research, vol. 127, pp. 259-270, 2015.

[8] M. Panteli and P. Mancarella, "The Grid: Stronger, Bigger, Smarter?: Presenting a Conceptual Framework of Power System Resilience," IEEE Power \& Energy Magazine, vol. 13, no. 3, pp. 58-66, 2015.

[9] Y. Wang, C. Chen, J. Wang, and R. Baldick, "Research on Resilience of Power Systems Under Natural Disasters - A Review," IEEE Trans. Power Syst., vol. 31, no. 2, pp. 1604-1613, Mar. 2016.

[10] M. Ouyang and L. Dueñas-Osorio, "Time-dependent resilience assessment and improvement of urban infrastructure systems," Chaos, vol. 22, p. 033122, Sept. 2012.

[11] M. Ouyang and L. Dueñas-Osorio, "Multi-dimensional hurricane resilience assessment of electric power systems," Struct. Safety, vol. 48, pp. 15-24, May 2014.

[12] D. Henry and J. Emmanuel Ramirez-Marquez, "Generic metrics and quantitative approaches for system resilience as a function of time," Rel. Eng. \& Syst. Safety, vol. 99, pp. 114-122, Mar. 2012.

[13] D. A. Reed, K. C. Kapur, and R. D. Christie, "Methodology for Assessing the Resilience of Networked Infrastructure", IEEE Syst. Journal, vol. 3, no. 2, pp. 174-180, May 2009.

[14] G. P. Cimellaro, A. M. Reinhorn, and M. Bruneau, "Framework for analytical quantification of disaster resilience," Engin. Struct., vol. 32, pp. 3639-3649, Nov. 2010. 
[15] M. Ouyang, L. Dueñas-Osorio, and X. Min, "A three-stage resilience analysis framework for urban infrastructure systems," Struct. Safety, vol. 36-37, pp. 23-31, May-Jul. 2012.

[16] M. Panteli, C. Pickering, S. Wilkinson, R. Dawson, and P. Mancarella, "Power System Resilience to Extreme Weather: Fragility Modelling, Probabilistic Impact Assessment, and Adaptation Measures," in IEEE Trans. Power Systems, Early Access, pp.1-10, Dec. 2016

[17] S. Espinoza, M. Panteli, P. Mancarella, and H. Rudnick, "Multi-phase assessment and adaptation of power systems resilience to natural hazards," Electric Power Systems Research, vol. 136, pp. 352-361, Jul. 2016.

[18] M. Belivanis and K. Bell, "Representative GB Network Model," 2011, Available: www.maths.ed.ac.uk/optenergy/NetworkData/reducedGB/, Last Accessed: September 2016

[19] M. Panteli, D. N. Trakas, P. Mancarella, and N. D. Hatziargyriou, "Boosting the Power Grid Resilience to Extreme Weather Events Using Defensive Islanding," IEEE Trans. Smart Grid, vol. 7, no. 10, pp. 29132922, Mar. 2016.

[20] National Grid PLC, "Electricity Ten Year Statement," UK, 2013.

[21] M. Kirsty and K. R. W. Bell, "Wind Related Faults on the GB Transmission Network," in proc: Probabilistic Methods Applied to Power Systems (PMAPS), Durham, UK, 2014, pp. 1-6.

[22] UK Met Office, "Beaufort wind force scale". Available: http://www.metoffice.gov.uk/guide/weather/marine/beaufort-scale

[23] European Centre for Medium-Range Weather Forecasts, "ERA Interim", Available: http://apps.ecmwf.int/datasets/data/interim-fulldaily/levtype $=$ sfc/

\section{BIOGRAPHIES}

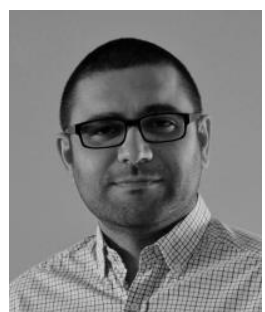

Mathaios Panteli (S'09, M'13) received the MEng degree in 2009 from Aristotle University of Thessaloniki, Greece, and the $\mathrm{PhD}$ degree in Electrical Power Engineering from The University of Manchester, UK in 2013, where he also worked as a Post-doctoral Research Associate for a few years. In September 2015, he joined University of Cyprus as a Research Associate and University of Nicosia, Cyprus, as an Adjunct Lecturer. He is currently a Lecturer in the Power and Energy Division at The University of Manchester. His main research interests include analysis and prevention of blackouts and risk and resilience assessment of power systems.

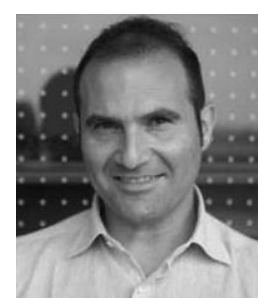

Pierluigi Mancarella (M'08-SM'14) received the $\mathrm{MSc}$ and $\mathrm{PhD}$ degrees in electrical energy systems from the Politecnico di Torino, Torino, Italy, in 2002 and 2006, respectively. $\mathrm{He}$ is currently Chair Professor of Electrical Power Systems at The University of Melbourne, Australia, and Professor of Smart Energy Systems at The University of Manchester, UK. His research interests include multienergy systems, power system integration of low carbon technologies, network planning under uncertainty, and risk and resilience of smart grids. Pierluigi is an Editor of the IEEE Transactions on Smart Grid, an Associate Editor of the IEEE Systems Journal, and an IEEE PES Distinguished Lecturer.

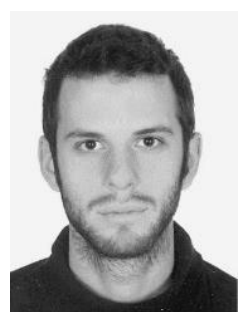

Dimitris N. Trakas (S'16) received his diploma in Electrical and Computers Engineering from National Technical University of Athens (NTUA), Greece in 2009, and the Master of Sciences in Energy Production and Management from NTUA in 2011. He is currently a Ph.D. student at Electric Power Division of NTUA. His research interests include decentralized control of power systems, resilience assessment and enhancement of power systems.

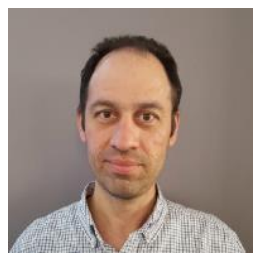

Elias Kyriakides (S'00, M'04, SM'09) received the B.Sc. degree from the Illinois Institute of Technology in Chicago, Illinois in 2000, and the M.Sc. and Ph.D. degrees from Arizona State University in Tempe, Arizona in 2001 and 2003 respectively, all in Electrical Engineering. He is currently an Associate Professor in the Department of Electrical and Computer Engineering at the University of Cyprus, and a founding member of the KIOS Research Center for Intelligent Systems and Networks. He served as the Action Chair of the ESF-COST Action IC0806 "Intelligent Monitoring, Control, and Security of Critical Infrastructure Systems" (IntelliCIS) (2009-2013). His research interests include synchronized measurements in power systems, security and reliability of the power system network, optimization of power system operation techniques, and the integration of renewable energy sources.

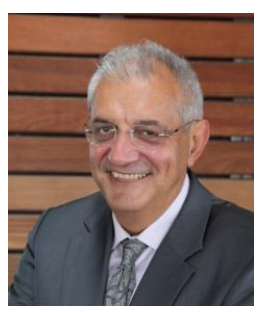

Nikos D. Hatziargyriou is Chairman and CEO of the Hellenic Distribution Network Operator. Since 1984 he is with the Power Division of the Electrical and Computer Engineering Department of the National Technical University of Athens and since 1995 he is full professor in Power Systems. From February 2007 to September 2012, he was Deputy CEO of the Public Power Corporation (PPC) of Greece, responsible for Transmission and Distribution Networks, island DNO and the Center of Testing, Research and Prototyping. He is Fellow Member of IEEE, past Chair of the Power System Dynamic Performance Committee, Distinguished member of CIGRE and past Chair of CIGRE SC C6 "Distribution Systems and Distributed Generation". He is cochair of the EU Technology and Innovation Platform on Smart Networks for Energy Transition. He is member of the Energy Committee of the Athens Academy of Science. He has participated in more than 60 R\&DD Projects funded by the EC and the industry, and has coordinated among others, the EU "CARE", "MORE CARE", "MERGE", "Microgrids" and "More Microgrids" projects. He is author of the book "Microgrids: Architectures and Control" and of more than 200 journal publications and 500 conference proceedings papers. 